\title{
COMMENTARY
}

\section{Bone marrow-derived mononuclear cell therapy in sepsis-induced acute respiratory distress syndrome: different insults, different effects!}

\author{
Paolo Pelosi ${ }^{1 *}$ and Yuda Sutherasan ${ }^{1,2}$ \\ See related research by Maron-Gutierrez et al., http://stemcellres.com/content/4/5/123
}

\begin{abstract}
Acute respiratory distress syndrome (ARDS) is one of the devastating sequelae of sepsis, and so far no specific promising pharmacotherapies have been proven to decrease mortality from it. Stem cell therapy is a novel therapy that can promote earlier and more effective remodeling and repair of damaged lung tissue. Bone marrow-derived mononuclear cells are an alternative stem cell therapy that is safely and easily administered on the day of harvesting and yields benefits in acute disease processes like ARDS. In a recent issue of Stem Cell Research and Therapy, Maron-Gutierrez and colleagues demonstrated that the effects of transfused bone marrow-derived mononuclear cells on lung mechanics, inflammation and mortality might be different in different septic ARDS models due to different insults.
\end{abstract}

Stem cell therapy may be a promising treatment for improving acute respiratory distress syndrome (ARDS) outcome. In a recent issue of Stem Cell Research and Therapy, Maron-Gutierrez and colleagues demonstrated the effect of bone marrow-derived mononuclear cell (BMDMC) transfusion after different insults resulting in extrapulmonary ARDS (ARDSexp) in terms of lung mechanics, lung inflammation and mortality [1].

Protective mechanical ventilation with low tidal volumes, moderate to high positive end expiratory pressure [2] and prone position [3] have been demonstrated to improve survival in severe ARDS. Mortality from severe ARDS is still high, however, ranging from 27 to $45 \%[4,5]$,

\footnotetext{
*Correspondence: ppelosi@hotmail.com

'IRCCS AOU San Martino-IST, Department of Surgical Sciences and Integrated Diagnostics, University of Genoa, Largo Rosanna Benzi 8, 16132, Genova, Italy Full list of author information is available at the end of the article
}

and increases for up to 5 years after the initial illness [6,7]. Despite extensive research on different pharmacologic drugs, no pharmacologic therapies have been proven to decrease mortality in severe ARDS.

Stem cell therapy aiming to reduce lung injury while maintaining the host immune response has been proposed as a novel potentially effective treatment for ARDS. Several experimental studies have demonstrated that stem cell therapy, including exogenous infusion and endogenous recruitment, can help in the remodeling and repair of damaged lung tissue $[8,9]$.

Mesenchymal stem/stromal cell therapies are widely implemented in experimental studies. However, they present some limitations, especially in acute disease, such as culture conditions being detrimental for cell transplantation, risk of contamination and unwanted immunological reactions [10]. BMDMCs represent an alternative stem cell therapy containing both hematopoietic and nonhematopoietic stem cells, such as mesenchymal stem/stromal cells $[8,9]$. They have been demonstrated to be safe, rapid and easy to administer on the day of harvesting and yield benefits in acute disease processes like ARDS [11]. The main therapeutic effects of BMDMCs, reported in different ARDS models, are: 1) prevention of lung inflammation, alveolar collapse, and interstitial edema; 2) repair of epithelial and endothelial cells; 3 ) improvement in lung elastance [12]; and 4) decreased collagen fiber content and cell apoptosis in lung and extrapulmonary organs [11]. These effects appear to have a greater effect in ARDSexp models, such as polymicrobial infection models (cecal ligation and puncture-induced sepsis) [13].

To test the effects and mechanisms of BMDMCs after different types of initial insult, Maron-Gutierrez and colleagues investigated their effects in murine models of ARDS induced by intraperitoneal Escherichia coli lipopolysaccharide (LPS) or cecal ligation and puncture (CLP; 
polymicrobial infection model) [1]. Endothelial cell activation plays an important role in sepsis through increased expression of adhesion molecules such as intracellular adhesion molecule (ICAM)-1. With regard to the different initial insults, BMDMC administration led to different effects on adhesion molecule mRNA expression. In the LPS injury model, BMDMC therapy increased ICAM-1 mRNA expression on day 7 , in agreement with results reported by a previous study [14]. In the CLP injury model, BMDMC therapy increased vascular cell adhesion molecule-1 mRNA expression on day 7. However, Zhang and colleagues [15] reported an association between ICAM-1 and CLP-induced sepsis. These data demonstrate that BMDMC therapy has distinct effects on lung inflammation during ARDS depending on the initial insult, even when the etiology is the same (extrapulmonary).

In both injury models, BMDMC therapy decreased the total cell count of neutrophils and macrophages in lung tissue over time. It also resulted in decreased interleukin$1 \beta$ mRNA expression, the cytokine mediating the activation of leucocyte recruitment and activation, which is consistent with the cell counts in lung tissue. However, an increase in the number of broncho-alveolar lavage fluid mononuclear cells was observed at day 3 in both the control and LPS injury groups treated with BMDMCs. BMDMC administration led to a significant decrease in collagen content in lung tissue, suggesting BMDMCs have a role in lung repair. Nevertheless, these changes in lung parenchyma were not associated with transforming growth factor- $\beta$ mRNA and protein expression in lung tissue, which means that BMDMC therapy decreases lung fibrosis through activation of pathways other than that related specifically to transforming growth factor- $\beta$. BMDMC administration resulted in an improvement of lung mechanics in terms of lung elastance and alveolar collapse compared with no BMDMC treatment. In the CLP model, BMDMCs improved mortality compared to the control group, but the authors were unable to demonstrate any difference in outcome between the two different types of initial insult. This might be explained by there being no deaths in the LPS group, likely due to differences in severity between the single insult in the LPS model and the multiple insults in the polymicrobial infection model as well as differences in immune mechanisms and cytokine responses.

The study by Maron-Gutierrez and colleagues is important since it provides additional knowledge on the potential beneficial role of BMDMCs in ARDSexp, showing that the degree of effect is related to the type of initial insult. Moreover, this therapeutic approach is shown to be clinically applicable because experiments were performed in animal models that can imitate human ARDS. Other studies are required to better define the optimal dose of BMDMCs, as well as the best effective route of administration, and timing in ARDS due to different insults.

\section{Conclusion}

BMDMCs are able to mitigate pulmonary inflammation, as well as decrease lung elastance, lung remodeling and fibrosis, resulting in lower mortality in ARDSexp experimental models. The benefits of BMDMCs depend on the type of initial insult as well as different effects on endothelial cell activation and adhesion molecules. Further research is needed to clarify these mechanisms and to examine this novel therapy in clinical trials.

\section{Abbreviations}

ARDS: Acute respiratory distress syndrome; ARDSex: extrapulmonary ARDS; BMDMC: Bone marrow-derived mononuclear cell; CLP: Cecal ligation and puncture; ICAM: Intracellular adhesion molecule; LPS: Lipopolysaccharide.

\section{Competing interests}

The authors declare that they have no competing interests.

\section{Author details}

'IRCCS AOU San Martino-IST, Department of Surgical Sciences and Integrated Diagnostics, University of Genoa, Largo Rosanna Benzi 8, 16132, Genova, Italy. ${ }^{2}$ Ramathibodi Hospital, Mahidol University, 270 RAMA VI road, Rajatewee,

Phayathai, Bangkok, Thailand.

\section{Published: 26 Nov 2013}

\section{References}

1. Maron-Gutierrez T, Silva JD, Cruz FF, Alegria S, Xisto DG, Assis EF, CastroFaria-Neto HC, Santos CCD, Morales MM, Rocco PRM: Insult-dependent effect of bone marrow cell therapy on inflammatory response in a murine model of extrapulmonary acute respiratory distress syndrome. Stem Cell Res Ther 2013, 4:123.

2. Putensen C, Theuerkauf N, Zinserling J, Wrigge H, Pelosi P: Meta-analysis: ventilation strategies and outcomes of the acute respiratory distress syndrome and acute lung injury. Ann Internal Med 2009, 151:566-576.

3. Gattinoni L, Taccone P, Carlesso E, Marini JJ: Prone position in acute respiratory distress syndrome: rationale, indications and limits. Am J Respir Crit Care Med 2013 [Epub ahead of print].

4. Hernu R, Wallet F, Thiollière F, Martin O, Richard JC, Schmitt Z, Wallon G, Delannoy B, Rimmelé T, Démaret C, Magnin C, Vallin H, Lepape A, Baboi L, Argaud L, Piriou V, Allaouchiche B, Aubrun F, Bastien O, Lehot JJ, Ayzac L, Guérin C: An attempt to validate the modification of the AmericanEuropean consensus definition of acute lung injury/acute respiratory distress syndrome by the Berlin definition in a university hospital. Intensive Care Med 2013, 39:2161-2170.

5. Force ADT, Ranieri VM, Rubenfeld GD, Thompson BT, Ferguson ND, Caldwell E, Fan E, Camporota L, Slutsky AS: Acute respiratory distress syndrome: the Berlin Definition. JAMA 2012, 307:2526-2533.

6. Esteban A, Frutos-Vivar F, Muriel A, Ferguson ND, Peñuelas O, Abraira V, Raymondos K, Rios F, Nin N, Apezteguía C, Violi DA, Thille AW, Brochard L, González M, Villagomez AJ, Hurtado J, Davies AR, Du B, Maggiore SM, Pelosi P, Soto L, Tomicic V, D'Empaire G, Matamis D, Abroug F, Moreno RP, Soares MA, Arabi $Y$, Sandi F, Jibaja M, et al: Evolution of mortality over time in patients receiving mechanical ventilation. Am J Respir Crit Care Med 2013, 188:220-230.

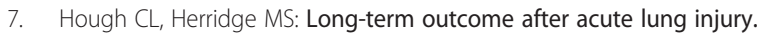
Curr Opin Crit Care 2012, 18:8-15.

8. Abreu SC, Antunes MA, Pelosi P, Morales MM, Rocco PR: Mechanisms of cellular therapy in respiratory diseases. Intensive Care Med 2011, 37:1421-1431.

9. Hayes M, Curley G, Ansari B, Laffey JG: Clinical review: Stem cell therapies for acute lung injury/acute respiratory distress syndrome - hope or hype? Crit Care 2012, 16:205.

10. Hayes M, Curley G, Laffey JG: Mesenchymal stem cells - a promising therapy for acute respiratory distress syndrome. F1000 Med Rep 2012, 4:2.

11. Araújo IM, Abreu SC, Maron-Gutierrez T, Cruz F, Fujisaki L, Carreira H Jr, Ornellas F, Ornellas D, Vieira-de-Abreu A, Castro-Faria-Neto HC, Muxfeldt Ab'Saber A, Teodoro WR, Diaz BL, Peres Dacosta C, Capelozzi VL, Pelosi P, 
Morales MM, Rocco PR: Bone marrow-derived mononuclear cell therapy in experimental pulmonary and extrapulmonary acute lung injury. Crit Care Med 2010, 38:1733-1741.

12. Prota LF, Lassance RM, Maron-Gutierrez T, Castiglione RC, Garcia CS, Santana MC, Souza-Menezes J, Abreu SC, Samoto V, Santiago MF, Capelozzi VL, Takiya CM, Rocco PR, Morales MM: Bone marrow mononuclear cell therapy led to alveolar-capillary membrane repair, improving lung mechanics in endotoxin-induced acute lung injury. Cell Transplant 2010, 19:965-971.

13. Ornellas DS, Maron-Gutierrez T, Ornellas FM, Cruz FF, Oliveira GP, Lucas IH, Fujisaki L, Oliveira MG, Teodoro WR, Capelozzi VL, Pelosi P, Morales MM, Rocco PR: Early and late effects of bone marrow-derived mononuclear cell therapy on lung and distal organs in experimental sepsis. Respir Physiol Neurobiol 2011, 178:304-314.

14. Kandasamy K, Sahu G, Parthasarathi K: Real-time imaging reveals endothelium-mediated leukocyte retention in LPS-treated lung microvessels. Microvasc Res 2012, 83:323-331.

15. Zhang H, Zhi L, Moochhala SM, Moore PK, Bhatia M: Endogenous hydrogen sulfide regulates leukocyte trafficking in cecal ligation and puncture-induced sepsis. J Leukoc Biol 2007, 82:894-905.

\section{$10.1186 /$ scrt354}

Cite this article as: Pelosi and Sutherasan: Bone marrow-derived mononuclear cell therapy in sepsis-induced acute respiratory distress syndrome: different insults, different effects!. Stem Cell Research \& Therapy 2013, 4:143 